\title{
Magnetic monopoles in a low energy world: emergency and fractionalization in natural and artificial spin ices
}

\author{
Afranio R. Pereira*, L. A. S. Mól, W. A. Moura-Melo \\ Departamento de Física, Universidade Federal de Viçosa, Viçosa, 36570-000, Minas Gerais, \\ Brazil \\ E-mail: apereiradufv.br
}

\begin{abstract}
Magnetic monopoles have not been found yet in particle accelerators or cosmic rays. On the other hand, some recent researches have shown that they may arise as emergent fractional quasiparticles inside natural and artificial spin ice materials, which are frustrated magnetic insulators. In this paper we discuss the theoretical and experimental results concerning these emergent monopoles and their strings in two-dimensional artificial spin ice systems. Indeed, these fractional objects were indirectly detected in the natural materials (which are three-dimensional systems) while they were directly observed in the artificial square lattice, confirming some previous theoretical predictions.
\end{abstract}

4th International Conference on Fundamental Interactions

August 1-7, 2010

Viçosa, Brazil

\footnotetext{
* Speaker.
} 
Magnetic monopoles have always been a curiosity for physicists, inspiring the imagination ever since Dirac's [W] observation that their existence could be reconciled with quantum mechanics. In the 1970s, there were searches going for them, and the most famous one was led by Cabrera [[]]. He reported to have found a monopole in his experiments but, despite extensive posterior searching, another monopole was never seen again. Indeed, in the experiments of high energy physics, no one has seen clear evidence for magnetic monopoles, leading us to believe that the Cabrera's experiment was spurious. In this work, we would like to talk about such elusive objects but not as a particle that falls from the sky or arises from the large particle accelerators. Our monopoles are rather concerned with the idea of emergence: they will show up as elementary quasi-particles in some exotic systems of low energy physics known as spin ice materials.

The fundamental question of a reductionist point of view is: what are the fundamental building blocks of the Universe? Maybe it is impossible to discover these deepest structures. What is more, maybe it does not matter what they are. The idea of emergency is that, maybe what we call reality is a spontaneous phenomenon, emerging like wave out of some unknowable cosmic medium. As a consequence, the laws that govern large-scale phenomena will not be deduced from the laws that govern tiny particles. Emergence is a familiar phenomenon in condensed matter (generally, a low, or even very low energy world). It is the branch of physics which studies systems of many particles in the condensed states (solid and liquid) and several of its theoretical developments were done with the help of quantum field theories. Solids and liquids sometimes play host to strange entities that bear little resemblance to the atoms and electrons making up the original substance. The large number of atoms and their strong interactions "conspire" to produce the emergence of quasiparticles with strange properties. Many kinds of emergent "creatures" live inside condensed matter materials, including phonons (vibrational waves in a crystal or superfluid), magnons in magnetic systems, excitons in semiconductors (electrical excitations) and also wave of charge called plasmons. There are also several topological excitations that behave like particles as vortices (in two-dimensional magnetic materials, superfluids and superconductors), skyrmions and so on. Since it is the quantum world, waves can be considered as particles, and vice-versa, so these excitations behave like a kind of emergent particle. Therefore, if quasiparticles are indistinguishable from real particles, a question arises: are that things we think as real electrons, photons and so on quasiparticles themselves, emerging out of some ubiquitous but undetectable cosmic stuff? It is a controversial idea. Indeed, in the conventional scenery (Standard Model $U(1) X S U(2) X S U(3)$ ), we consider that fermions and gauge bosons are fundamental and untouchable; we introduce by hand a fundamental $U(1)$ gauge field and anti-commuting fermion fields. Indeed, until recently, physicists did not know of any collective modes that behave like gauge bosons and fermions. However, things have changed and due to the advances over the last twenty years, we now know how to

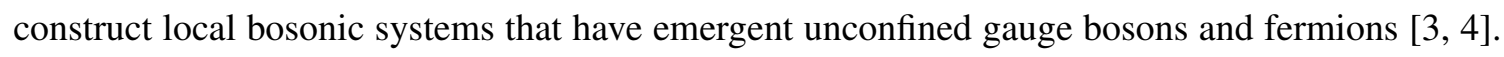
This local bosonic model, known as String Net Condensation (SNC), is an unification of gauge fields ("light") and fermions; light is a fluctuation of nets of condensed strings of arbitrary sizes while fermions are identified to the ends of condensed strings. Therefore, it can be shown that gauge fields and fermions are emergent. They exist because our vacuum happens to have string net condensation. However, it is still missing to show that chiral fermions and gravity (space-time) are also emergent.

Our aim here is less ambitious. Following the idea of emergency, we would like to consider 
a condensed matter system in which the phenomenon of "fractionalization" plays an important role. This occurrence is associated with the fact that, in some materials, the important excitations are quasi-particles that carry only a fraction of the properties of the original particles (atoms, electrons) that constitute the compound. It is very common in one-dimensional materials (polyacetylene, nanotubes etc) and is often tied to topological defects [可]. One of the most interesting example is the behavior of electrons in a quantum scale wire (an one-dimensional system); in this situation electrons behave as divisible particles. They can break into two smaller particles, called spinons and holons. A spinon is a charge-neutral quasiparticle that carries only the spin of the electron while a holon carries only its charge. The breaking-apart effect only happens when a lot of electrons are competing for not a lot of space[目]. In two spatial dimensions the only confirmed case of fractionalization is the involvement of quasi-particles with one-third of an electron's charge in the fractional quantum Hall effect in strong magnetic fields. On the other hand, several materials are now candidate to be a two-dimensional quantum spin liquid, which is a state of matter with a disordered ground-state. For a quantum spin liquid, the excited states may be also spinons. Depending on the type of spin liquid, the spinons may obey Fermi or Bose statistics and there may or may not be an energy gap. Furthermore, these spinons can not live by themselves but are generally accompanied by gauge fields, just as electrons are always accompanied by electromagnetic gauge fields [प, [0]]. Considering two-dimensional Heisenberg antiferromagnets with spin-1/2, some works $[\mathbb{8}, \mathbf{Q}]$ have suggested that the merons forming a two-core skyrmion are also spinons. All these lower-dimensional examples are emergent phenomenon, where new particles and fields emerge at low-energy scales but are totally absent in the Hamiltonian (or action) that describe the original system.

In three dimensions, fractionalization is not so common [ए2, प]]. The first real example were discovered only recently and happens in a material known as spin ice. Castelnovo et.al. [U]] have shown how the famous magnetic monopole may emerge inside these materials. Three-dimensional spin-ice materials are an exotic class of crystalline solid having the pyrochlore structure in which magnetic rare-earth ions form a lattice of corner-sharing tetrahedra. To minimize the spin-spin interaction energy, the ice rules are manifested: two spins point inward and two spins point outward on each tetrahedron. This is a magnet that behaves like the water ice (justifying its name), presenting also a phenomenon so called geometrical frustration: the spins are constrained by geometry to form a configuration which the pairwise interaction energy cannot be simultaneously minimized for all constituents. The authors of Ref.[[प]]] then observed that, in this world of magnetic dipoles (spins) organized in a spin ice configuration, the violation of the ice rule generate two excitations carrying oppositive magnetic charge, like magnetic monopoles. Indeed, such "monopoles" can be separated from one another without further violations of local neutrality by flipping a chain of adjacent spins. In this process a tensionless and observable "string" of spins pointing from the positive to the negative charge is created. Note that, in this case, the magnetic charge does not need to obey the Dirac condition [四]. This follows from the monopole being attached to a Dirac string, which has to be unobservable. By contrast, the string soup characteristic of spin ice at low temperature makes the strings energetically unimportant, although they are observable and therefore not quantized. Calculations [ए]] and experiments by Bramwell et.al. [[13] based on Wien's theory of electrolytes show that the magnetic charge is about 8000 smaller than the Dirac fundamental charge. In addition to the interesting experiments of Bramwell et.al. [[13], several other recent 


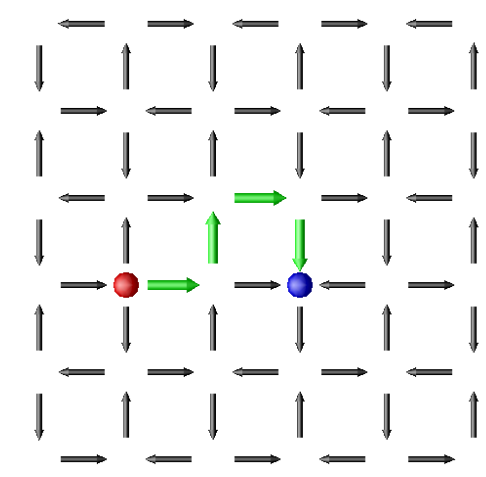

Figure 1: (Color online) Schematic view of the system. The black arrows represents the background configuration according to the ground-state one expects for $h<0.444 a$. The green arrows and the spheres represents the excitations above the ground-state. The different colors of the spheres represent the oppositive poles (north and south) while the green arrows are the string of dipoles connecting the monopoles.

experiments [[4], ㅁ5, 미] have reported the observation of the magnetic charge and current of these monopoles in spin ice materials; simulations also support these ideas [[17, [18]. However, to turn the research of monopoles into a proper applied science, it will be necessary to ask if the basic ideas of dipole fractionalization [ $\mathbb{W}, \mathbb{2}]$ ] that give an usual spin-ice material its special properties can be realized in other magnetic settings. One of the most promising candidates for accomplishing that, is the artificial version of spin ices recently produced by Wang et al. [प्स]. In this system, elongated magnetic nano-islands are regularly distributed in a two-dimensional $(2 d)$ square lattice. The longest axis of the islands alternate its orientation pointing in the direction of the two principal axis of the lattice [10]. The magnetocrystalline anisotropy of Permalloy (the magnetic material commonly used to fabricate artificial spin ice) is effectively zero, so that the shape anisotropy of each island forces its magnetic moment to align along the largest axis, making the islands effectively Ising-like.

These artificial frustrated magnets are objects of intense study in recent years [1], [20, 20], [2], [23, [24, [25, [26, 227, [28]. In addition to the square lattices, kagome[27], hexagonal and brickwork geometries have also been produced [25]. Since their geometries are determined lithographically, lattice symmetry and topology can be directly controlled, allowing experimental investigation of a vast set of important theoretical models of statistical physics [29]. The possible existence of these excitations in artificial and controllable systems would be great because they could be directly observed with the modern experimental techniques. In fact, very recently, a direct observation of a kind of magnetic monopole defects and their motion (with the aid of magnetic force microscopy) in an artificial kagome spin ice was reported by Ladak et al. [27]. Before such an observation, we have proposed that magnetic monopoles and their strings could also arise in the original $2 d$ square lattice [22, [26]. Our results have now been experimentally confirmed by interesting experiments [B]] in which the authors identify the small localized departures from the ground state as elementary excitations of the system (monopoles and strings), at frequencies that follow a Boltzmann law. 
Here, we study an arrangement of dipoles similar to that experimentally investigated in Ref. [미] (see Fig. W, where we show the ordered background and a monopole-antimonopole pair connected by an energetic string). Actually, we will consider a more general case: a modified system as proposed by Möller and Moessner[[ए]] in which the arrangement of Ref.[एप] is a particular case. Such modification consists of introducing a height offset $h$ between islands pointing along the two different directions [20, [23]. This system was not fabricated yet although it is a structured material to be considered in a near future. Our object here is to revise the main properties of these systems for some values of $h$, including $h=0$. In our scheme, however, the magnetic moment ("spin") of the island is replaced by a point dipole at its center. In each site $\left(x_{i}, y_{i}, z_{i}\right)$ of the modified square lattice two spin variables are defined: $\vec{S}_{x(i)}$ with components $S_{x}= \pm 1, S_{y}=0, S_{z}=0$ located at $\vec{r}_{x}=\left(x_{i}+1 / 2, y_{i}, h\right)$, and $\vec{S}_{y(i)}$ with components $S_{x}=0, S_{y}=/ p m 1$ and $S_{z}=0$ at $\vec{r}_{y}=\left(x_{i}, y_{i}+1 / 2,0\right)$. Therefore, in a lattice of volume $L^{2}=l^{2} a^{2}$ ( $a$ is the lattice spacing) one gets $2 \times l^{2}$ spins. Representing the spins of the islands by $\vec{S}_{i}$, which can assume either $\vec{S}_{x(i)}$ or $\vec{S}_{y(i)}$, then the artificial spin ice is described by the following Hamiltonian

$$
H_{S I}=D a^{3} \sum_{i \neq j}\left[\frac{\vec{S}_{i} \cdot \vec{S}_{j}}{r_{i j}^{3}}-\frac{3\left(\vec{S}_{i} \cdot \vec{r}_{i j}\right)\left(\vec{S}_{j} \cdot \vec{r}_{i j}\right)}{r_{i j}^{5}}\right],
$$

where $D=\mu_{0} \mu^{2} / 4 \pi a^{3}$ is the coupling constant of the dipolar interaction. The sum is either over all $l^{2}\left(2 l^{2}-1\right)$ pairs of spins in the lattice for the case with open boundary conditions (OBC) or over all spins and their images for the case with periodic boundary conditions (PBC). In the system with PBC a cut-off radius is introduced at $L / 2$.

We now present a summary of the main results obtained from Hamiltonian above considering this compound at zero temperature [ㄹ, [26]. For the case of our interest, $h<0.444 a$, we got the ground state configuration of the system in a square lattice, showing that it is twofold degenerate. Such result was also obtained experimentally very recently[B]] for the case $h=0$. Indeed, the ground state was experimentally very difficult to access using the effective thermodynamics of rotating-field demagnetization protocols, because the energy barriers to thermal equilibrium are extremely large. However, the authors of Ref.[B]] studied an as-fabricated sample that approaches the ground state very closely. If one considers the vorticity in each plaquette, assigning a variable $\sigma=+1$ and -1 to clockwise and anticlockwise vorticities respectively, the ground state looks like a checkerboard, with an antiferromagnetic arrangement of the $\sigma$ variable. Of course, the ground state clearly obeys the ice rule. The most elementary excitation is related to the inversion of a single spin (dipole) to generate a localized pair of defects. This is the $3-i n, 1-$ out state in a particular vertex and the $3-$ out, $1-$ in state in its adjacent vertex. In principle, these defects could be separated without further violation of the ice rule. Really, in our previous paper [22], we have numerically shown that these defects behave as a monopole-antimonopole pair since their interaction (see Fig. ( ( $\mathbb{Z}$ )) follows a $d=3$ Coulomb law $q / R$, where $q$ measures the strength of the interaction and $R$ is the distance between the poles. However, we have also pointed out that they could not appear as effective low-energy degrees of freedom in the $2 d$ square spin ice because the background antiferromagnetic order in the ground state confines them [122]. Actually, there are additional excitations not present in the usual three-dimensional ( $3 d$ ) spin ice [W], namely, energetic one-dimensional strings of dipoles (resultant spins at each vertex along a line of adjacent 
vertices) that terminate in monopoles and antimonopoles. Such string excitations cost an energy equal to $b$ times their length $X$, where $b$ is the string tension. When the temperature $T$ of the system is near absolute zero, the shortest path length connecting the monopoles gives the potential energy. Therefore, the total cost of a monopole-antimonopole pair separated by a distance $R$ is the sum of the usual Coulombic term roughly equal to $q / R$, and a term roughly equal to $b X$ resulting from the string joining the monopoles (there is, of course, also a constant term of energy associated to the creation of a pair). The ordering causes an anisotropy in the system making the monopoles interaction highly dependent of the direction in which the monopoles are separated in the crystal plane [ [R6]. This anisotropy is manifested in both, the Coulomb and linear terms the potential in such a way that we explicitly write [22, 26]

$$
E(h, R, X)=q(h, \phi) / R+b(h, \phi) X+c(h)
$$

where $\phi$ is an angle that the line joining the monopole defects makes with the $x$-axis of the array and $c$ is related to the creation energy of a pair of defects. The first term is the Coulomb law and the magnetic field propagates in the three-dimensional space, even for the pure two-dimensional case with $h=0$. The second term is caused by the background (the magnetic islands forming the system) due to the two distinct topologies of the spins obeying the ice rule in the vertices [एT]. Unfortunately, in two dimensions, these two different topologies are not degenerate (in contrast to the natural $3 d$ spin ices). This fact causes a string with tension $b$, which connects the monopoles. Numerically, for the case $h=0$, which can be compared with experiments, we got $q(0,0) \approx-3.87 D a$, $b(0,0) \approx 9.75 D / a$ while $q(0, \pi / 3) \approx-4.01 D a, b(0, \pi / 3) \approx 10.14 D / a ; c(0)$ is approximately $23 D$. for both cases. On the other hand, for $h \rightarrow 0.444 a$, the system is almost isotropic with $q \approx-3.8 D a$ and $b \approx 0.5 D / a$. Note that in the limit $h \rightarrow 0.444 a$, the string tension almost vanishes and the monopoles tend to become more separated and free [23]. In Fig. B] we show the constant $c$ as a function of $h$. For $h=0$, the small localized departures from the ground state (monopole pair and its string) were experimentally identified [BO] as elementary excitations of the system, following the Boltzmann law with $b \approx 10 D / a$ and $q / a+b a+c=E(0, a, a) \approx 30 D$ (the creation energy), which are very closely to our theoretical predictions (in our works $E(0, a, a) \approx 29 D$ ).

The Coulomb coupling $q$ is associated to the magnetic charge $Q$ of a magnetic pole in the artificial material as follows: $Q(h, \phi)= \pm \sqrt{4 \pi|q(h, \phi)| / \mu_{0}}$. Using typical values of a fabricated sample, $a \approx 320 \mathrm{~nm}, D \approx 2 \times 10^{-19} \mathrm{~J}$ etc, the mean value of the magnetic charge is on the order of $2.1 \mu / a$, which is about 100 times smaller than the Dirac fundamental charge but about 80 times bigger than the magnetic charge in the natural three-dimensional spin ices. At low temperatures, there is insufficient thermal energy to create long strings (with length $X$ larger than one lattice spacing) and so, the monopoles are bound together tightly in pairs. On the other hand, as the temperature is increased, the average separation between the constituents of a pair also increases, which means that larger strings may become present in the system. Indeed, experiments have now identified and cataloged the monopoles and their strings, showing "large" separations and several shapes of strings in the lattice [B]]. For instance, monopoles are found separated by distances on the order of $1200 \mathrm{~nm}$ with string still bigger. A simple argument given in Ref.[22] shows that the string tension should vanish above a critical temperature given by $T_{c} \approx b a / k_{B}$, where $k_{B}$ is the Boltzmann constant. Therefore, the monopoles should become completely free at high tempera- 


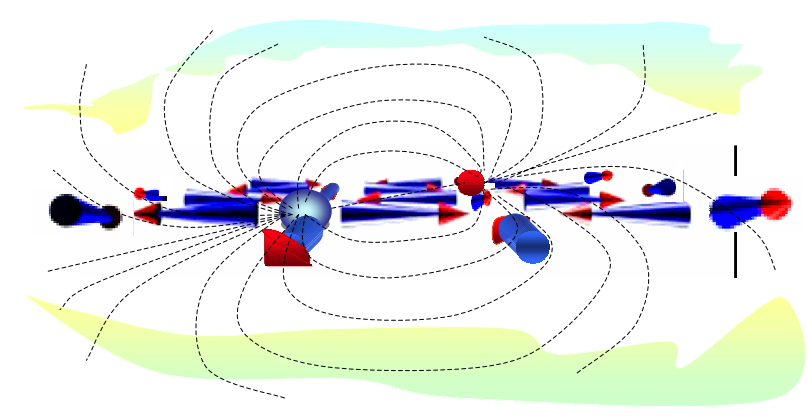

Figure 2: (Color online) Lateral view of an artificial $2 d$ spin ice $(h=0)$ containing a pair of monopole defects in two vertices (blue and red circles). Their interaction follows the Coulomb law and propagates in the $3 d$ space. However, the medium in which they live (the magnetic islands) create an energetic string, connecting them. Such string should be vanished by temperature effects [22] or by making a three-dimensional

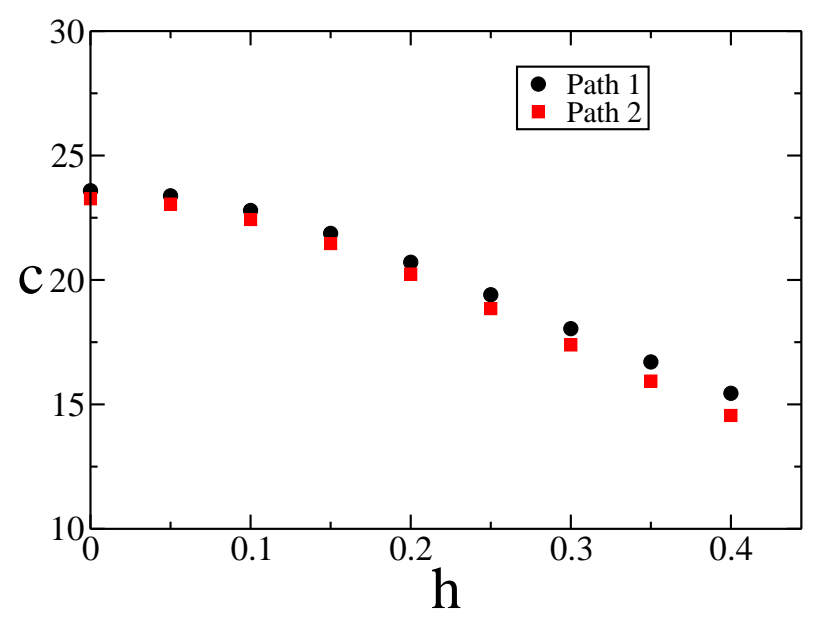

Figure 3: (Color online) In this figure we show the constant $c$ as a function of $h$ for two different string paths as shown in Ref. [ㅈ]. Note that as the height-off set is increased the creation energy is reduced.

tures. Monte Carlo calculations are being prepared to see this possibility and must be published further. At low temperatures these poles are confined like quarks in the quantum chromodynamics and, nevertheless, these systems should provide a good place to experimentally study, by using direct observation, the effects of confinements. The shape of strings and its dynamics may lead to some insights to the problems of quark confinement inside protons, neutrons etc.

As commented above, the fundamental magnetic charge of an excitation in the array of ferromagnetic nano-islands reads $Q \approx \sqrt{4 \pi|q| / \mu_{0}}$, which gives $\approx 2.1 \mu / a$ for $a=320 \mathrm{~nm}$. Such a charge can even be tuned continuously by changing the lattice spacing. For smaller $a$, the charge increases. In the experiments of Ref.[30], the lattice spacing is $400 \mathrm{~nm}$ and, therefore, the magnetic charge is smaller. Before concluding, it is also important to analyze the behavior of the string tension as the lattice spacing and the height offset $h$ are varied in the system. The string tension for the artificial system built in Ref.[[प⿴囗十] $](h=0)$ is approximately given by $b \approx 10 D$. Therefore, it is necessary a relatively large amount of energy to separate the monopoles by one lattice spacing, regardless of how far apart they are. Consequently, at low temperature, there is insufficient thermal 
energy to create long strings, and so the "monopoles" would be bound together tightly in pairs. The string tension can be artificially reduced by increasing the parameter $a(b \propto 1 / a)$. However, it has also the effect of decreasing the magnetic charge. A way to reduce $b$, without affecting $Q_{M}$ is increasing the height offset $h$. Indeed, the tension decreases rapidly as $h$ increases and almost vanishes for $h=0.444 a$, but the magnetic charge is practically unaffected [26]. Therefore, a possible deconfined phase for all temperatures would live in systems with $h \approx 0.444 a$ as pointed in Refs. [22], 26]. Thermal fluctuations are also other effect that should decrease the string tension [22]. Although these artificial systems are athermal, demagnetization field protocols can provide effective thermodynamics[3]], which should be included in the problem of string configurational entropy[22, [26]. Once the deconfinement completely realizes, the question of technological applications of this system is relevant. For instance, learning how to move the magnetic monopoles around would be of importance towards technologies involving magnetic analogous of electric circuits.

\section{Acknowledgments}

The authors thank CNPq, FAPEMIG and CAPES (Brazilian agencies) for financial support.

\section{References}

[1] P.A.M. Dirac, Proc. Roy. Sos. (London) A133, 60 (1931).

[2] B. Cabrera, Phys. Rev. Lett. 48, 1378 (1982).

[3] X.-G. Wen, Phys. Rev. Lett. 88, 11602 (2002).

[4] X.-G. Wen, Quantum Field Theory of Many-Body Systems, Oxford University Press, New York, (2004); and references therein.

[5] J. Goldstone and F. Wilczek, Phys. Rev. Lett.47, 986 (1981).

[6] B.J. Kim, H. Kos, E. Rotenberg, S.-J. Oh, H. Eisaki, N. Motoyama, S. Uchida, T. Tohyama, S. Maekawa, Z.-X. Shen, and C. Kim, Nature Phys. 2, 397 (2006).

[7] P.A. Lee, N. Nagaosa, and X.-G. Wen, Rev. Mod. Phys. 78, 17 (2006).

[8] G. Baskaran, Phys. Rev. B68, 212409 (2003).

[9] A.R. Moura, A.R. Pereira, and A.S.T. Pires, Phys. Rev. B 75, 014431 (2007).

[10] P.A. Lee, Science 321, 1306 (2008)

[11] C. Castelnovo, R. Moessner, and L. Sondhi, Nature 451, 42 (2008).

[12] Z. Nussinov, C.D. Batista, B. Normand, and S.A. Trugman, Phys. Rev. B 75, 094411 (2007).

[13] S.T. Bramwell, S.R. Giblin, S. Calder, R. Aldus, D. Prabhakaran, and T. Fennell, Nature 461, 956 (2009).

[14] T. Fennell, P.P. Deen, A.R. Wildes, K. Schmalzl, D. Prabhakaran, A.T. Boothroyd, R.J. Aldus, D.F. McMorrow, and S.T. Bramwell, Science 326, 415 (2009).

[15] D.J. P. Morris, D.A. Tennant, S.A. Grigera, B. Klemke, C. Castelnovo, R. Moessner, C. Czternasty, M. Meissner, K.C. Rule, J.-U. Hoffmann, K. Kiefer, S. Gerischer, D. Slobinsky, and R.S. Perry, Science 326, 411 (2009). 
[16] H. Kadowaki, N. Doi, Y. Aoki, Y. Tabata, T.J. Sato, J.W. Lynn, K. Matsuhira, and Z. Hiroi, J. Phys. Soc. Jpn. 78, 103706 (2009).

[17] L.D.C. Jaubert and P.C.W. Holdsworth, Nature Phys. 5, 258 (2009).

[18] C. Castelnovo, R. Moessner, and S.L. Sondhi, Phys. Rev. Lett. 104, 107201 (2010).

[19] R.F. Wang, C. Nisoli, R.S. Freitas, J. Li, W. McConville, B.J. Cooley, M.S. Lund, N. Samarth, C. Leighton, V.H. Crespi, and P. Schiffer, Nature 439, 303 (2006).

[20] G. Möller and R. Moessner, Phys. Rev. Lett. 96, 237202 (2006).

[21] X. Ke, J. Li, C. Nisoli, P. E. Lammert, W. McConville, R.F. Wang, V.H. Crespi, and P. Schiffer, Phys. Rev. Lett. 101, 037205 (2008).

[22] L.A. Mól, R.L. Silva, R.C. Silva, A.R. Pereira, W.A. Moura-Melo, and B.V. Costa, J. Appl. Phys. 106, 063913 (2009).

[23] G. Möller and R. Moessner, Phys. Rev. B 80, 140409(R) (2009).

[24] H. Zabel, A. Schumann, W. Westphalen, and A. Remhof, Acta Phys. Pol. A 115, 59 (2009).

[25] J. Li, X. Ke, S. Zhang, D. Garand, C. Nisoli P. Lammert, V.H. Crespi, and P. Schiffer, Phys. Rev. B 81, 092406 (2010).

[26] L.A.S. Mól, W.A. Moura-Melo, and A.R. Pereira, Phys. Rev. B 82, 054434 (2010).

[27] S. Ladak, D.E. Read, G.K. Perkins, L.F. Cohen, and W.R. Brandford, Nature Phys. 6, 359 (2010).

[28] A. Remhof, A. Shumann, A. Westphalen, H. Zabel, N. Mikuszeit, E.Y. Vedmedenko, T. Last, and T. Kunze, Phys. Rev. B 77, 134409 (2008).

[29] R. Baxter, Exactly Solved Models in Statistical Physics(Academc Press, New York, 1982).

[30] J.P. Morgan, A. Stein, S. Langridge, and C. H. Marrows, Nature Phys. doi:10.1603/1.3224870, (2010).

[31] C. Nisoli, R. Wang, J. Li, W.F. McConville, P.E. Lammert, P. Schiffer, and V.H. Crespi, Phys. Rev. Lett.98, 217203 (2007). 